\title{
Toxicology and prescribed medication histories among people experiencing fatal illicit drug overdose in British Columbia, Canada
}

\author{
Alexis Crabtree MD PhD, Emily Lostchuck MD MSc, Mei Chong MSc, Aaron Shapiro MSc PhD, \\ Amanda Slaunwhite PhD
}

Cite as: CMAJ 2020 August 24;192:E967-72. doi: 10.1503/cmaj.200191

See related article at www.cmaj.ca/lookup/doi/10.1503/cmaj.201618

\begin{abstract}
BACKGROUND: Since 2015, illicit drug overdose has been one of British Columbia's most pressing public health issues. Our objective was to assess prescription history in the context of postmortem toxicology among people who had a fatal illicit drug overdose in $\mathrm{BC}$.
\end{abstract}

METHODS: Toxicology results from drug overdose deaths involving 1 or more illicit drugs, as identified by the BC Coroners Service in 2015-2017, were linked to the prescription drug histories of individuals as recorded in BC's PharmaNet database for a descriptive analysis. Substances identified in toxicology were considered prescribed if the individual had an active dispensation for a matching medication within 60 days before overdose.

RESULTS: There were 2872 deaths from illicit drug toxicity during the study period; 1789 (62.3\%) were closed cases with toxicology results available. In $85.5 \%$ of cases, 1 or more opioids were found to be relevant to death. Prescribed opioids in the absence of nonprescribed opioids were detected in only $2.0 \%$ of cases, and $6.7 \%$ had a combination of prescribed and nonprescribed opioids. Among those with 1 or more nonprescribed opioids, $78.5 \%$ had fentanyl or fentanyl analogues detected. Medications used in opioid agonist therapy (methadone and buprenorphine) were found to be relevant to death in $7.4 \%$ of cases, with methadone (130 cases) much more common than buprenorphine $<5$ cases). Stimulants were detected in $70.6 \%$ of cases.

INTERPRETATION: Our data show a high prevalence of nonprescribed fentanyl and stimulants, and a low prevalence of prescribed opioids detected on toxicology in people who died from illicit drug overdose. These results suggest that strategies to address the current overdose crisis in Canada must do much more than target deprescribing of opioids. n British Columbia, overdose has been responsible for a devastating loss of life. ${ }^{1}$ From 2014 to 2016, fatal drug overdose contributed to a decrease of 0.38 years $^{2}$ in the life expectancy of $B C$ residents, and since 2015, fatal overdoses have been the province's leading cause of unnatural death. ${ }^{3}$ The rising number of overdose deaths led the $\mathrm{BC}$ government to declare a public health emergency in April 2016. ${ }^{4}$ Despite the tireless efforts of first responders, many of whom are themselves people with lived experience of drug use, and the rapid implementation of new medical and outreach services for people at risk of overdose, the rates of deaths attributed to illicit drug overdose remain unacceptably high in BC and exceed rates in other Canadian provinces. ${ }^{1,5,6}$

The opioid overdose crisis was initially primarily attributed to the misuse of prescription opioids. However, despite decreases in the prescribing of opioids in $\mathrm{BC}$, the death rate from illicit drug toxicity has increased more than fourfold, from 7.8/100 000 in 2014 to
30.9/100 000 in 2018., ${ }^{3,7}$ This unprecedented increase in overdoserelated deaths has been primarily attributed to the introduction of fentanyl into the illicit drug supply. ${ }^{3,9}$ From 2012 to 2018, the proportion of deaths from illicit drug overdose in $\mathrm{BC}$ in which fentanyl was detected rose from $4 \%$ to $87 \% .^{3}$ Similar increases in fentanylattributed deaths have been observed in Alberta, the United States and, to a lesser extent, Ontario. ${ }^{10,11}$ Similarly, stimulants have been increasingly implicated in deaths from drug overdose. ${ }^{12}$

With health professional organizations introducing guidelines to reduce prescribing of opioids and other controlled substances, understanding the relative contribution of prescribed substances and illicitly obtained substances to overdose deaths is key to developing effective programs to reduce overdose mortality. We used a linked population-based data set of overdose deaths to compare toxicology with active prescriptions at death to characterize the role of prescribed drugs in deaths from illicit drug overdose. While 
the role of illegal fentanyl in the overdose crisis is well known, this study allows assessment at the individual level of the prescription status of substances that can be prescribed or obtained by other means, including opioids for pain, opioid agonist therapy, benzodiazepines and stimulants.

\section{Methods}

\section{Data sources and definitions}

The BC Provincial Overdose Cohort is a linked administrative database of medical, prescribing and postmortem data on people who have had a fatal or nonfatal overdose in the province of BC. A full description of case definitions and data sources for the Provincial Overdose Cohort is available from MacDougall and colleagues. ${ }^{13}$

For this analysis, cases were defined as all people who have had a fatal illicit drug overdose (at least 1 substance relevant to death was illegally produced or diverted), as determined by the BC Coroners Service. Investigations by the BC Coroners Service involve collection of postmortem samples for comprehensive toxicological and other laboratory testing, autopsy and review of available medical records. The coroner responsible for the case determines the substances that are relevant to death based on the levels found in blood and tissue, pharmacologic knowledge and other available data about the case. Data were available for the years 2015-2017. The sample was restricted to closed cases for which toxicology results were available.

Individuals were linked to their prescription medication histories using PharmaNet, a comprehensive electronic medical record system that contains information on dispensations from community pharmacies for all residents of BC. All included BC Coroners Service cases were linkable. We assessed for the presence of an active prescription within 60 days before overdose (with the period of active prescription defined as the day of medication dispensation until the supply end date as estimated by the pharmacist). Individuals were also linked to their medical histories to assess comorbidities in the year before overdose using the Discharge Abstract Database (for hospital admissions), Medical Services Plan billing data (for outpatient physician visits), and the National Ambulatory Care Reporting System (for emergency department visits), and using algorithms described by Smolina and colleagues. ${ }^{9}$ Demographic information (sex and age) was drawn from the BC Ministry of Health Provincial Client Roster. All closed cases were linkable to these 4 data sets, and there was no missing demographic information.

Toxicology results were available to the $\mathrm{BC}$ Provincial Overdose Cohort only for substances determined by the BC Coroners Service to have been relevant to the death (i.e., not incidentally detected). Substances were considered prescribed if there was an exact match between prescription and toxicology (e.g., hydromorphone on prescription and toxicology) or a correspondence between a prescription and a metabolite on toxicology (e.g., fentanyl on prescription and norfentanyl on toxicology). Heroin was identified through detection of 6-monoacetylmorphine (a metabolite).

The following classes of medication were assessed: opioids, benzodiazepines and Z-drugs, stimulants, gabapentinoids, antidepressants and antipsychotics. A full list of the medications included in these classes is available in Appendix 1, available at www.cmaj.ca/lookup/suppl/doi:10.1503/cmaj.200191/-/DC1.

\section{Data analysis}

Descriptive data analysis was conducted by the authors using SAS Enterprise Guide 7.1. Cell sizes less than 5 were suppressed in accordance with the information sharing agreement of the Provincial Overdose Cohort.

\section{Ethics approval}

As this analysis was conducted as part of the surveillance and epidemiology functions of the BC Centre for Disease Control, ethics review was not required.

\section{Results}

\section{Study population}

A total of 2872 deaths from illicit drug overdose occurred in $B C$ in 2015-2017. Of these, 1789 (62.3\%) were closed cases with toxicology results available. In comparison, during the same period, 105 deaths occurred that were attributed to prescription drugs; the number is subject to change pending closure of investigations (Andrew Tu, BC Coroners Service, Burnaby, BC: personal communication, 2020). There were no clinically important differences

Table 1: Characteristics of people who died from illicit drug overdose in British Columbia, 2015-2017

Characteristic

No. $(\%)$ of people $n=1789$

Demographic characteristics

Age group, $y r$

$\leq 30$ $435(24.3)$

31-49 859 (48.0)

$\geq 50$ 495 (27.7)

Sex, male $1440(80.5)$

\section{Active prescription for}

Opioid for pain

149 (8.3)

Benzodiazepine or Z-drug

235 (13.1)

Antidepressant

$272(15.2)$

Antipsychotic

$47(2.6)$

Stimulant

Gabapentin

155 (8.7)

Antiepileptic

$57(3.2)$

\section{Diagnoses in the past year}

Cardiovascular disease

Respiratory disease

Substance use disorder

$599(33.5)$

Alcohol use disorder

Schizophrenia, bipolar disorder or personality

disorder

Depression, anxiety or adjustment disorder $†$

*With or without co-occurring depression, anxiety or adjustment disorder. tWithout co-occurring schizophrenia, bipolar or personality disorder. 
between closed and open cases (data available from author on request). Closed cases were more likely to have had overdose occur in 2015 and 2016 (96.8\% and 91.6\% of cases were closed, respectively) than in 2017 ( $34.7 \%$ were closed). A total of $80.5 \%$ of the people who died from illicit drug overdose during the study period were male, and the median age was 40 years (Table 1 ). In total, $33.5 \%$ of the people who died had a diagnosis of substance use disorder in the year before overdose.

Most deaths from illicit drug overdose in BC involved opioids (Table 2). Among cases with no opioids found relevant to death, the most common substances were cocaine (47.1\%), methamphetamine $(39.0 \%)$, amphetamines $(34.0 \%)$ and alcohol (27.0\%).

\section{Prescribed and nonprescribed opioids}

A total of $120 / 1789$ (6.7\%) had a combination of prescribed and nonprescribed opioids relevant to death, and 36/1789 (2.0\%) had only prescribed opioids found relevant to death (Table 2). Thus, of all the cases of deaths from illicit drug overdose in $\mathrm{BC}$ from 2015 to $2017,1374 / 1789$ (76.8\%) were found to have only nonprescribed opioids relevant to death. Of the individuals with 1 or more nonprescribed opioids found relevant to death, fentanyl and fentanyl analogues were found in more than twice as many cases as heroin (78.5\% v. 33.1\%) (Table 3).

\section{Methadone and buprenorphine}

Methadone was found to be relevant to death more often than buprenorphine (130 cases v. $<5$ cases) but was still only relevant in $130(7.3 \%)$ of the illicit drug deaths for which toxicology was available. Of the cases for which methadone was relevant, 50 $(38.5 \%)$ had no history of it being prescribed within 60 days before overdose, and 43 (33.1\%) had no history of methadone prescribed within 365 days before overdose. Of people with an active methadone prescription on the day of overdose $(n=38)$, $20(52.6 \%)$ were prescribed a daily dose of less than $60 \mathrm{mg}$, and only $12(31.6 \%)$ had doses of $80 \mathrm{mg}$ or greater. ${ }^{14}$ All of the people $(<5)$ whose death was related to buprenorphine had an active prescription within 60 days before overdose.

Table 2: Prescription status* of medication relevant to death among illicit drug overdose deaths in British Columbia, 2015-2017

\section{Prescription status}

Any opioid relevant to death

Only prescribed opioids

Prescribed and nonprescribed opioids

Only nonprescribed opioids

Stimulants relevant to death

Only prescribed stimulants

Prescribed and nonprescribed stimulants

Only nonprescribed stimulants

Antidepressants relevant to death

Only prescribed antidepressants

Prescribed and nonprescribed antidepressants

Only nonprescribed antidepressants

Benzodiazepines and Z-drugs relevant to death

Only prescribed benzodiazepines or Z-drugs

Prescribed and nonprescribed benzodiazepines

Only nonprescribed benzodiazepines or Z-drugs

Antipsychotics relevant to death

Only prescribed antipsychotics

Prescribed and nonprescribed antipsychotics

Only nonprescribed antipsychotics

Gabapentinoids relevant to death

Only prescribed gabapentinoids

Prescribed and nonprescribed gabapentinoids

Only nonprescribed gabapentinoids
No. (\%) of deaths by substance class and prescription status $n=1789$

$1530(85.5)$

$36(2.0)$

$120(6.7)$

$1374(76.8)$

$1263(70.6)$

$<5(<0.3)$

$10(0.6)$

$1251(70.0)$

$122(6.8)$

$82(4.6)$

$13(0.7)$

$27(1.5)$

$107(6.0)$

$35(2.0)$

$5(0.3)$

$67(3.7)$

$46(2.6)$

$38(2.2)$

0

$8(0.4)$

$29(1.6)$

24 (1.3)

0

$5(0.3)$
Substance class-related deaths, \%

$$
\begin{gathered}
1530(100) \\
36(2.4) \\
120(7.8) \\
1374(89.8) \\
1263(100) \\
<5(<0.4) \\
10(0.8) \\
1251(99.0) \\
122(100) \\
82(67.2) \\
13(10.7) \\
27(22.1) \\
107(100) \\
35(32.7) \\
5(4.7) \\
67(62.6) \\
46(100) \\
38(82.6) \\
0 \\
8(17.4) \\
29(100) \\
24(82.8) \\
0 \\
5(17.2) \\
\hline
\end{gathered}
$$


Table 3: Presence of fentanyl or fentanyl analogues and heroin among cases of fatal illicit drug overdose in British Columbia, 2015-2017

No. $(\%)$ of cases

\section{Opioid class relevant to death}

Fentanyl or fentanyl analogue*

Heroin

No fentanyl analogue or heroin

*3-Methylfentanyl, acetylfentanyl, carfentanil or furanylfentanyl.

Total among all illicit drug deaths $n=1789$

$1174(65.6)$

494 (27.6)

$409(22.9)$
Total among cases with

$\geq 1$ nonprescribed opioid $n=1494$

$1173(78.5)$

$494(33.1)$

$115(7.7)$

\section{Benzodiazepines and Z-drugs}

Only 107 (6.0\%) fatal cases had benzodiazepines or Z-drugs relevant to death. In 67 (62.6\%) of these cases, benzodiazepines and Z-drugs had not been prescribed within 60 days before overdose (Table 2). The most commonly detected nonprescribed benzodiazepines were alprazolam and diazepam (detected in 42 [62.7\%] and 9 [13.4\%], respectively, of cases with only nonprescribed benzodiazepines or Z-drugs).

\section{Other classes of medication}

Other classes of medication that were assessed were stimulants, gabapentinoids, antidepressants and antipsychotics (Table 2). In total, $1263(70.6 \%)$ of cases of fatal drug overdose had stimulants relevant to death. Among these people, very few had prescriptions for these medications $(0.1 \%)$. Absolute numbers of cases with gabapentinoids, antidepressants and antipsychotics relevant to death were low $(1.6 \%, 6.8 \%, 2.6 \%$, respectively).

\section{Interpretation}

We examined the postmortem toxicology of confirmed illicit drug-related deaths in relation to comprehensive prescription histories. Of cases with opioids found relevant to death, only $2.4 \%$ of cases had prescribed-only opioids found relevant in toxicology. A further $7.8 \%$ of cases had a combination of prescribed and nonprescribed opioids detected. Most people (90\%) with opioids relevant to death had not been prescribed these substances. Of the cases with any nonprescribed opioids, fentanyl or fentanyl analogues were found relevant to death in $78.5 \%$ of cases. Opioid agonist therapy was relevant to only $7.4 \%$ of total illicit drug deaths, of which $38.5 \%$ of cases involving methadone had no active prescription at the time of death. Stimulants were found to be relevant to $70.6 \%$ of illicit drug deaths and, of these, less than $1.0 \%$ of people had been prescribed the stimulants detected. Finally, of the small number of cases in which benzodiazepines or Z-drugs were detected, more than half $(62.6 \%)$ of these individuals had not obtained these substances through a prescription.

In BC, prescription-only overdose deaths have decreased as a relative percentage of all overdose deaths, with illegal overdose deaths 7-30 times as common as prescription-only overdose deaths in $2015-2017^{3}$ and representing more than $96 \%$ of drug- related deaths during the study period. Our findings underscore the declining role of prescription opioids and heroin in the overdose crisis and the rise of synthetic opioids and stimulants. Previous studies comparing toxicology and prescribing have found a much higher contribution of prescription drugs to overdose death, ranging from $29 \%$ to $67 \%$, and further described oxycodone as the most prevalent opioid found on toxicology, with fentanyl accounting for less than $15 \%$ of deaths. ${ }^{15-17}$ These studies generally occurred more than 5 years ago before the emergence of fentanyl and are not directly comparable because they excluded nonprescription opioid overdose deaths. We found prescribed opioids to be relevant to only $2.4 \%$ of opioid-related illicit drug deaths in BC between 2015 and 2017. Similar research from Ontario showed a decline in active opioid prescriptions associated with opioid overdose deaths from 2013 to $2016 .{ }^{7}$

Recent studies suggest that deaths from prescription opioids have been stable from 2009 to 2016, despite the precipitous rise in opioid overdose deaths. ${ }^{3,11,18}$ The decreasing role of prescription opioids suggests that efforts at deprescribing opioids, and guidelines encouraging nonopioid management of chronic pain have led to reduced opioid prescriptions. Our findings suggest that prescribing policies are insufficient to address the current overdose crisis in Canada and additional strategies are needed. Physicians should be encouraged to practise patient-centred opioid prescribing. The risk of harms from these medications (including the harms of starting new long-term opioid therapy and the harms of continuing therapy instead of offering alternative therapies with better evidence of efficacy) must be balanced with the potential harms of nonconsensual discontinuation of opioids for long-term users, including increased pain, risk of suicide and risk of transition to the toxic illicit drug supply. ${ }^{19-22}$

Stimulants, overwhelmingly nonprescribed, were relevant to $71 \%$ of the illicit drug deaths in our sample, in keeping with the rise of unprescribed stimulant use across North America ${ }^{12}$ and increasing methamphetamine use in $\mathrm{BC} .^{23}$ The relation between stimulant use, opioid and other substance use, and risk of death is complex. Stimulants may be used to manage the sedation caused by opioids or benzodiazepines; opioids may be used to bring on sleep or manage anxiety among people using stimulants; and some people may choose to use them together because of preference for their joint effects. ${ }^{24}$ The risk associated 
with these types of use will be different depending on tolerance to the substances, with people who do not regularly use opioids (and therefore have lower tolerance than regular users) considered to be at higher risk of overdose when they do use opioids, including opioid-adulterated stimulants. Unintentional use may also contribute to our findings - it is unknown to what extent illegal stimulants are adulterated or contaminated with opioids, or vice versa. For people who have a stimulant use disorder there is no definitive gold standard of treatment, although a body of literature emphasizes the value of psychosocial interventions and an emerging role for sustained-release dexamphetamine. ${ }^{25-28}$

The use of methadone, and more recently buprenorphine, as opioid agonist therapy is known to be a safe and effective treatment for opioid use disorder. ${ }^{29-32}$ We found that nonprescribed methadone was present in less than $2.0 \%$ of total illicit drug deaths, and that the methadone prescribed to people who fatally overdosed was generally at low doses that are less effective at preventing overdose. ${ }^{14}$ Buprenorphine was present in less than $0.1 \%$ of illicit drug deaths, consistent with its known safety profile as a partial agonist with lower risk of respiratory depression than methadone. ${ }^{33-37}$ This may also reflect its poorer retention rate and lower uptake in $\mathrm{BC} .{ }^{35,38}$ Our findings support the continued emphasis on decreasing barriers to medically supervised opioid agonist therapy, to provide a safer alternative to contaminated illegal drugs and diverted methadone. Supervised dosing, which is time-consuming and has limited evidence to support its use, ${ }^{39}$ should be carefully considered as part of efforts to lower barriers to opioid agonist therapy. Use of nonprescribed methadone can be an important alternative for people who do not wish to access clinical opioid agonist therapy services and who wish to reduce reliance on the toxic drug supply. ${ }^{40}$ Thus, supporting peer-based organizations and harm-reduction agencies can provide an opportunity to help people more safely manage nonmedically supervised methadone use.

We found nonprescribed benzodiazepines to be more commonly associated with illicit drug deaths than prescribed benzodiazepines. With decreasing prescription of benzodiazepines in Canada and increasing detection globally of novel illegal benzodiazepines, attention should be given to supporting people with benzodiazepine use disorder. ${ }^{8,41-43}$ This is particularly salient given that alprazolam was the most commonly detected nonprescribed benzodiazepine in our sample. Alprazolam is rarely prescribed in BC (only $2 \%$ of dispensations) but was the most commonly purchased benzodiazepine in the US in an analysis of online drug cryptomarkets ( $>50 \%$ of sales). ${ }^{44,45}$ Contamination of illegal opioids with benzodiazepines is also an increasing concern. ${ }^{46} \mathrm{An}$ important area for future study is the contribution of polypharmacy to overdose risk in populations using nonprescribed medications and, in particular, the contribution of stimulants and sedatives to overdose mortality.

\section{Limitations}

A limitation of this analysis is that we were unable to identify the sources of medications that were consumed but not prescribed. For example, illicit methadone, whether diverted or imported, may have contributed to some of the fatal cases in which methadone was detected. Diversion or illegal importation is also an issue for other classes of medication. It is possible that substances found on toxicology but not dispensed in the community could have been the result of provision of samples at physicians' offices; consumption of previously prescribed medication; provision of medication by first responders, in acute care settings or in prison; or contamination or adulteration of other illegal drugs. An additional limitation is the use of 6-monoacetylmorphine to detect heroin in toxicology. Since this molecule is relatively unstable and prone to degrade during transport, we expect some underestimation of heroin use in the sample. This study examines a period of rapid change in the illegal drug market; caution should therefore be exercised in generalizing to other provinces and to the present day.

\section{Conclusion}

Among people who died from illicit drug overdose in BC in 2015$2017,85.5 \%$ had opioids relevant to death on toxicology; of these, both prescribed-only opioids (2.4\%) and a combination of prescribed and nonprescribed opioids (7.8\%) were relatively rare. Stimulants were commonly found relevant to death (70.6\%) and overwhelmingly nonprescribed. Few deaths (7.4\%) involved opioid agonist therapy. Of the small number of deaths involving benzodiazepines (6.0\% of illicit drug deaths), most (62.6\%) were nonprescribed. Our findings highlight that overdose is more than an opioid prescribing problem and requires a multifaceted response beyond prescribing and the medical system.

\section{References}

1. Special Advisory Committee on the Epidemic of Opioid Overdoses. National report: opioid-related harms in Canada Web-based report. Ottawa: Public Health Agency of Canada; 2019.

2. Ye X, Sutherland J, Henry B, et al. At-a-glance - Impact of drug overdoserelated deaths on life expectancy at birth in British Columbia. Health Promot Chronic Dis Prev Can 2018;38:248-51.

3. Illicit drug toxicity deaths in BC: January 1, 2010-April 30, 2020. Burnaby (BC): British Columbia Coroners Service; 2020. Available: www2.gov.bc.ca/assets/ gov/birth-adoption-death-marriage-and-divorce/deaths/coroners-service/ statistical/illicit-drug.pdf (accessed 2020 May).

4. Provincial health officer declares a public health emergency [news release]. $B C$ Government News 2016 Apr. 14. Available: https://news.gov.bc.ca/releases /2016HLTH0026-000568 (accessed 2020 Jan. 9).

5. Kolla G, Strike C. 'It's too much, I'm getting really tired of it': overdose response and structural vulnerabilities among harm reduction workers in community settings. Int J Drug Policy 2019;74:127-35.

6. Kennedy MC, Boyd J, Mayer S, et al. Peer worker involvement in low-threshold supervised consumption facilities in the context of an overdose epidemic in Vancouver, Canada. Soc Sci Med 2019;225:60-8.

7. Gomes T, Khuu W, Martins D, et al. Contributions of prescribed and nonprescribed opioids to opioid related deaths: population-based cohort study in Ontario, Canada. BMJ 2018;362:k3207.

8. Crabtree A, Rose $\mathrm{C}$, Chong $\mathrm{M}$, et al. Effects of the new prescribing standards in British Columbia on consumption of opioids and benzodiazepines and $z$ drugs. Can Fam Physician 2019;65:e231-7.

9. Smolina K, Crabtree A, Chong M, et al. Patterns and history of prescription drug use among opioid-related drug overdose cases in British Columbia, Canada, 2015-2016. Drug Alcohol Depend 2019;194:151-8.

10. Fischer B, Vojtila L, Rehm J. The 'fentanyl epidemic' in Canada - some cautionary observations focusing on opioid-related mortality. Prev Med 2018;107:109-13.

11. Data brief: opioid related overdose deaths among Massachusetts residents. Boston: Massachusetts Department of Health; 2018. Available: www.mass. gov/files/documents/2018/02/14/data-brief-overdose-deaths-february-2018. pdf (accessed 2020 Jan. 9).

12. Kariisa M, Scholl L, Wilson N, et al. Drug overdose deaths involving cocaine and psychostimulants with abuse potential - United States, 2003-2017. MMWR Morb Mortal Wkly Rep 2019;68:388-95. 
13. MacDougall L, Smolina K, Otterstatter M, et al. Development and characteristics of the provincial overdose cohort in British Columbia, Canada. PLoS One 2019;14:e0210129.

14. Provincial Opioid Use Disorder Treatment Guideline Committee. A guideline for the clinical management of opioid use disorder. Vancouver: British Columbia Centre on Substance Use and Victoria: BC Ministry of Health; 2017. Available: www.bccsu.ca/wp-content/uploads/2017/06/BC-OUD-Guidelines_June2017.pdf (accessed 2020 May).

15. Hall AJ, Logan JE, Toblin RL, et al. Patterns of abuse among unintentional pharmaceutical overdose fatalities. JAMA 2008;300:2613-20.

16. Madadi $P$, Hildebrandt D, Lauwers AE, et al. Characteristics of opioid-users whose death was related to opioid-toxicity: a population-based study in Ontario, Canada. PLoS One 2013;8:e60600.

17. Hirsch A, Proescholdbell SK, Bronson W, et al. Prescription histories and dose strengths associated with overdose deaths. Pain Med 2014;15:1187-95.

18. Seth P, Rudd RA, Noonan RK, et al. Quantifying the epidemic of prescription opioid overdose deaths. Am J Public Health 2018;108:500-2.

19. Dowell D, Haegerich TM, Chou R. CDC guideline for prescribing opioids for chronic pain - United States, 2016. JAMA 2016;315:1624-45.

20. Kertesz SG. Turning the tide or riptide? The changing opioid epidemic. Subst Abus 2017;38:3-8.

21. Currow DC, Phillips J, Clark K. Using opioids in general practice for chronic non-cancer pain: an overview of current evidence. Med J Aust 2016;205:334-5.

22. Opioids and methamphetamine: a tale of two crises. Lancet 2018;391:713.

23. Findings from the 2018 BC Harm Reduction Client Survey. Vancouver: BC Centre for Disease Control; 2018. Available: www.bccdc.ca/resource-gallery/Documents/ Statistics\%20and\%20Research/Statistics\%20and\%20Reports/Overdose/20181221 _BCCDC\%20Knowledge\%20Update_2018\%20BC\%20Harm\%20Reduction\%20 Survey.pdf (accessed 2020 Jan. 9)

24. Ellis MS, Kasper ZA, Cicero TJ. Twin epidemics: the surging rise of methamphetamine use in chronic opioid users. Drug Alcohol Depend 2018;193:14-20.

25. Fischer B, Blanken P, Da Silveira D, et al. Effectiveness of secondary prevention and treatment interventions for crack-cocaine abuse: a comprehensive narrative overview of English-language studies. Int J Drug Policy 2015;26:352-63.

26. Dutra L, Stathopoulou G, Basden SL, et al. A meta-analytic review of psychosocial interventions for substance use disorders. Am J Psychiatry 2008;165: 179-87.

27. Lee NK, Rawson RA. A systematic review of cognitive and behavioural therapies for methamphetamine dependence. Drug Alcohol Rev 2008;27:309-17.

28. Nuijten M, Blanken $P$, van de Wetering B, et al. Sustained-release dexamfetamine in the treatment of chronic cocaine-dependent patients on heroin-assisted treatment: a randomised, double-blind, placebo-controlled trial. Lancet 2016;387: 2226-34

29. Mattick RP, Breen C, Kimber J, et al. Buprenorphine maintenance versus placebo or methadone maintenance for opioid dependence. Cochrane Database Syst Rev 2014;(2):CD002207.

30. Mattick RP, Breen C, Kimber J, et al. Methadone maintenance therapy versus no opioid replacement therapy for opioid dependence. Cochrane Database Syst Rev 2009;(3):CD002209.
31. Lawrinson P, Ali R, Buavirat A, et al. Key findings from the WHO collaborative study on substitution therapy for opioid dependence and HIV/AIDS. Addiction 2008;103:1484-92.

32. Ward J, Hall W, Mattick RP. Role of maintenance treatment in opioid dependence. Lancet 1999;353:221-6

33. Bell JR, Butler B, Lawrance A, et al. Comparing overdose mortality associated with methadone and buprenorphine treatment. Drug Alcohol Depend 2009;104:73-7.

34. Ma J, Bao YP, Wang RJ, et al. Effects of medication-assisted treatment on mortality among opioids users: a systematic review and meta-analysis. Mol Psychiatry 2019;24:1868-83.

35. Sordo L, Barrio G, Bravo MJ, et al. Mortality risk during and after opioid substitution treatment: systematic review and meta-analysis of cohort studies. BMJ 2017;357:j1550.

36. Volpe DA, McMahon Tobin GA, Mellon RD, et al. Uniform assessment and ranking of opioid mu receptor binding constants for selected opioid drugs. Regul Toxicol Pharmacol 2011;59:385-90.

37. Boas RA, Villiger JW. Clinical actions of fentanyl and buprenorphine. The significance of receptor binding. Br J Anaesth 1985;57:192-6.

38. BC Opioid Substitution Treatment System: performance measures 2014/2015-2015/2106. Victoria: Office of the Provincial Health Officer; 2017. Available: www2.gov.bc.ca/assets/gov/health/about-bc-s-health-care-system/ office-of-the-provincial-health-officer/reports-publications/special-reports/bc-ost -system-measures-14-15-and-15-16.pdf (accessed 2020 Jan. 9).

39. Saulle R, Vecchi S, Gowing L. Supervised dosing with a long-acting opioid medication in the management of opioid dependence. Cochrane Database Syst Rev 2017;4:CD011983.

40. Harris M, Rhodes T. Methadone diversion as a protective strategy: the harm reduction potential of 'generous constraints.' Int J Drug Policy 2013;24:e43-50.

41. Manchester KR, Lomas EC, Waters L, et al. The emergence of new psychoactive substance (NPS) benzodiazepines: a review. Drug Test Anal 2018;10:37-53.

42. Pan-Canadian trends in the prescribing of opioids and benzodiazepines 2012 to 2017. Ottawa: Canadian Institute for Health Information; 2018. Available: www. cihi.ca/sites/default/files/document/opioid-prescribing-june2018-en-web.pdf (accessed 2020 Jan. 9)

43. Soyka M. Treatment of benzodiazepine dependence. N Engl J Med 2017;376: 1147-57.

44. Cunliffe J, Decary-Hetu D, Pollak TA. Nonmedical prescription psychiatric drug use and the darknet: a cryptomarket analysis. Int J Drug Policy 2019;73:263-72.

45. Health Sector Information, Analysis and Reporting Division Integrated Analytics: Community and Cross Sector. 2017/18 British Columbia Controlled Prescription Drug Atlas. Victoria: British Columbia Ministry of Health; 2018. Available: www2.gov. bc.ca/assets/gov/health/conducting-health-research/bc-controlled-prescription -drug-atlas-2017_2018-final.pdf (accessed 2020 Jan. 9).

46. Payer, DE, Young MM, Maloney-Hall B, et al. Adulterants, contaminants and cooccurring substances in drugs on the illegal market in Canada: an analysis of data from drug seizures, drug checking and urine toxicology. Ottawa: Canadian Centre on Substance Use and Addiction; 2020. Available: www.ccsa.ca/sites/ default/files/2020-04/CCSA-CCENDU-Adulterants-Contaminants-Co-occurring -Substances-in-Drugs-Canada-Report-2020-en.pdf (accessed 2020 July 24).

\section{Competing interests: None declared.}

This article has been peer reviewed.

Affiliations: School of Population and Public Health (Crabtree, Lostchuck), University of British Columbia; BC Centre for Disease Control (Chong); Department of Pathology and Laboratory Medicine (Shapiro, Slaunwhite), University of British Columbia; BC Provincial Toxicology Centre (Shapiro, Slaunwhite), Vancouver, BC

Contributors: Alexis Crabtree conceived of the study, and Aaron Shapiro, Amanda Slaunwhite, Mei Chong and Emily Lostchuck provided additional analytic direction. Mei Chong conducted the analyses. Alexis Crabtree, Emily Lostchuck and Amanda Slaunwhite drafted the manuscript and Mei Chong and Aaron Shapiro provided substantive review. All of the authors gave final approval of the version to be published and agreed to be accountable for all aspects of the work.

Data sharing: The data used in this study are not for further distribution under the terms of the information sharing agreement J16-145 that governs the Provincial Overdose Cohort however, researchers can apply for access to the original Provincial Overdose Cohort database at Population Data BC.

Acknowledgements: The authors thank members from the Provincial Overdose Cohort team (Bin Zhao, Wenqi Gan) and Prescribing Patterns Working Group (Kate Smolina, Tim Chu, Andrew Pacey, Christopher Mill, Christian Schütz, Yumian Hu, Jessica Moe, Frank Scheuermeyer Kari Harder, Keith Ahamad, Aamir Bharmal,
Scott Carlson, Niknaz Kahnamoui) for their guidance and input into this project. Data for this publication was provided by the $\mathrm{BC}$ Coroners Service, the BC Emergency Health Services, the $\mathrm{BC}$ Drug and Poison Information Centre, the BC Ministry of Health (PharmaNet, Medical Services Plan, the National Ambulatory Care Reporting System and the BC Discharge Abstract Database) and emergency departments in Interior, Island and Northern Health Authorities. All inferences, opinions and conclusions drawn are those of the authors, and do not reflect the opinions or policies of the data stewards.

Accepted: June 5, 2020

Correspondence to: Alexis Crabtree, alexis.crabtree@bccdc.ca 